\title{
« Reader, attend » : Robert Burns et l'art de la pointe
}

"Reader, attend": Robert Burns's Cutting Edge

\section{Yann Tholoniat}

\section{(2) OpenEdition}

\section{Journals}

Édition électronique

URL : http://journals.openedition.org/etudesecossaises/951

DOI : 10.4000/etudesecossaises.951

ISSN : 1969-6337

Éditeur

UGA Éditions/Université Grenoble Alpes

\section{Édition imprimée}

Date de publication : 25 avril 2015

Pagination : 67-86

ISBN : 978-2-84310-296-7

ISSN : 1240-1439

\section{Référence électronique}

Yann Tholoniat, « «Reader, attend »: Robert Burns et l'art de la pointe », Études écossaises [En ligne],

17 | 2015, mis en ligne le 25 avril 2016, consulté le 15 mars 2021. URL : http://

journals.openedition.org/etudesecossaises/951; DOI : https://doi.org/10.4000/etudesecossaises.951 


\section{«Reader, attend»: Robert Burns et l'art de la pointe}

Le dix-huitième siècle est le grand siècle de l'épigramme. C'est en 1776 que paraît la première édition non censurée de l'Anthologie palatine; c'est aussi l'époque des bons mots, et des mots féroces, comme celui de Voltaire contre Fréron («L'autre jour au fond d'un vallon, / un serpent mordit Jean Fréron. / Que croyez-vous qu'il arriva? / Ce fut le serpent qui creva»). Les épigrammes (à l'origine, une inscription brève et poétique imitée du latin) se diversifient et prennent diverses formes: exergue, histoire drôle, improvisation, dédicace, conseil... Leur nature les associe traditionnellement avec le franc-parler. Le poète romain Martial, lu, apprécié et défendu par Burns, revendiquait dans la préface à son recueil d'épigrammes «l'allègre franchise des mots, qui est la langue de l'épigramme» («lascivam verborum veritatem, id est epigrammaton linguam»). L'Écosse a sa propre tradition de l'épigramme, illustrée entre autres par George Buchanan (1506-1582) ${ }^{1}$. Une autre tradition de formules brèves et mémorables provient de la Bible, dont les formules gnomiques, les sentences, les proverbes (en réponse auxquels Blake compose les fulgurants «Proverbs of Hell ${ }^{2}$ », publiés dans Marriage of Heaven and Hell en 1794) imprègnent les discours des prédicateurs. Burns témoigne de son appréciation des citations qu'il aime à glaner de livre en livre :

I like to have quotations for every occasion. They give one's ideas so pat, and save one the trouble of finding expression adequate to one's feelings. I think it is one of the greatest pleasures attending a poetic genius, that we can give our woes, cares, joys, loves, etc., an embodied form in verse, which, to me, is ever immediate ease ${ }^{3}$.

1. Burns cite George Buchanan dans sa correspondance (Letters 1, p. 236 et 2, p. 73). Son ami John Moore avait écrit un roman, Zeluco, qui mettait en scène le poète humaniste.

2. Sur ces «Proverbs of Hell», voir notamment Porée (1995).

3. Lettre du 14 janvier 1788 à Agnes Mclehose (Letters 1, p. 207). 
À John Moore, il relate sa joie à la lecture et à l'imprégnation d'un recueil de bons mots : «I had met with a collection of letters by the wits of Queen Anne's reign, and I pored over them most devoutly ${ }^{4} . »$ Son anthologie personnelle est stratégique : «I pick up favourite quotations, and store them in my mind as ready armour, offensive or defensive, amid the struggle of this turbulent existence ${ }^{5}$.»

Dans son œuvre poétique, hormis «Tam o' Shanter», «The Vision» et «The Twa Dogs», ses poèmes dépassent rarement une centaine de vers. Dans le corps du poème, il travaille selon l'unité de sens formé par la strophe, au sein de laquelle il place fréquemment une maxime ou une formule ciselée à la pointe des rimes. Burns est un adepte du «fine remarkin'» («The Holy Fair», v. 49), et des formules comme «Pleasures are like poppy spread» («Tam o’ Shanter», v. 59) ont marqué les esprits. Certaines ont été adoptées par des artistes ultérieurs, comme "The best-laid schemes o' mice an 'men / Gang aft agley» ("To a Mouse», v. 39-40), reprise par John Steinbeck comme titre de son roman Of Mice and Men (1937), ou la chanson «Ae Fond Kiss», reprise par Ken Loach, pour le titre de son film sorti en 2004.

Parmi la variété des formes brèves, Burns privilégie les épitaphes et les épigrammes. Les deux formes constituent des commentaires, mais les contextes diffèrent. Ces «écritures sur» — un texte, une personne, défunte ou vivante - manifestent le désir de Burns d'aller à la rencontre ou à l'encontre des autres. La forme se situe au croisement de plusieurs genres et possède une grande diversité métrique et thématique. Funèbres, votives, épidictiques, érotiques, ironiques, ésotériques (Saïd, Trédé et Le Boullouec, 1997, p. 351), de l'humour au pathétique, tous les tons sont représentés, comme dans la satura traditionnelle, et tous les styles, du dépouillement au maniérisme. Ce qui caractérise l'épitaphe et l'épigramme, c'est qu'il s'agit de poésies de circonstances. Plusieurs de ces pièces brèves comportent dans le titre des éléments relatifs à l'occasion de leur composition («at», «on», «to», «Epigram on Said Occasion») ${ }^{6}$. Burns se vante de cette muse à l'humeur variable: «Poesy was still a darling walk for my mind, but 'twas only the humour of the hour ${ }^{7}$.»

4. Lettre du 2 août 1787 à John Moore (Letters 1, p. 141).

5. Lettre du 6 décembre 1792 à Mrs Dunlop (Letters 2, p. 165).

6. Burns les insère dans sa correspondance en expliquant les circonstances d'écriture, comme «To Dr Maxwell, on Miss Jessy Staig's recovery», dans une lettre à George Thomson : «Maxwell, if merit here you crave, / That merit I deny: / You save fair Jessy from the grave! / AN ANGEL could not die.- (lettre à George Thomson de septembre 1794, in Letters 2, p. 310). La lettre suivante, adressée à Mrs Dunlop, reprend la même épigramme, en y adjoignant une autre, «On Mr Walter Riddell, Esq. ».

7. Lettre du 2 août 1787 à John Moore (Letters 1, p. 141). 


\section{Les épitaphes}

L'épitaphe est une forme brève mise en exergue sur une pierre tombale. Texte isolé par définition, elle fonctionne par évocation : en peu de mots, il faut dire beaucoup. Il est impossible de résumer la vie d'une personne en deux ou trois lignes, et c'est un art difficile de graver dans la pierre : idéalement, l'épitaphe est un art du portrait sublimé sous une forme qui n'est pas sans rappeler, par sa brièveté, la forme du haiku. Traditionnellement, l'épitaphe, souvent en distique élégiaque, comporte une interpellation au passant et met en avant, brièvement, les qualités de la personne ${ }^{8}$. L'épitaphe comporte aussi parfois une dimension morale d'exemple et d'avertissement, prenant la forme d'un embrayeur.

«A Bard's Epitaph», qui clôt le recueil de 1786, contient la plupart de ces caractéristiques. De façon subtile, le poète-locuteur interpelle le passant qui se présenterait sous les traits de : «a whim-inspired fool» (v. 1), «a bard of rustic song» (v. 7), autrement dit, un passant qui serait lui aussi «a Heaven-taught ploughman» et un alter ego («frater», v. 11). Le jeu se double du paradoxe qui consiste pour le locuteur à composer sa propre épitaphe et de parler de soi alors qu'il est encore vivant. L'anaphore «Is there» (combinée à d'autres anaphores : «owre» aux vers 2 et 3 , «and» aux vers 5 et 6), qui interroge l'auditoire supposé de façon lancinante, débouche dans chaque strophe sur une réponse affirmative implicite, en y ajoutant une dimension pathétique :

Is there a whim-inspired fool,

Owre $^{\circ}$ fast for thought, owre hot for rule, $\quad{ }^{\circ}$ over/too

Owre blate ${ }^{\circ}$ to seek, owre proud to snool ${ }^{\circ}, \quad{ }^{\circ}$ shy, tamely submit

Let him draw near;

And owre this grassy heap sing dool ${ }^{\circ}$,

And drap ${ }^{\circ}$ a tear.

${ }^{\circ}$ sadly/lament

${ }^{\circ}$ drop

Is there a bard of rustic song,

Who, noteless, steals the crowds among,

That weekly this area throng,

$\mathrm{O}$, pass not by!

But, with a frater ${ }^{\circ}$-feeling strong, obrother-feeling

Here, heave a sigh.

8. L'épitaphe qui termine «Elegy on Captain Matthew Henderson», très courte, est paradigmatique : «Stop, passenger! my story's brief, / And truth I shall relate, man; / I tell nae common tale o' grief, / For Matthew was a great man.» 
Is there a man, whose judgment clear

Can others teach the course to steer,

Yet runs, himself, life's mad career,

Wild as the wave,

Here pause - and, thro' the starting tear,

Survey this grave. (v. 1-18)

La dernière strophe, admonestation envers cet autre/poète capable d'embrasser le microcosme et le macrocosme (v. 26-27), conclut sur une moralité du juste milieu :

Reader, attend - whether thy soul

Soars Fancy's flights beyond the pole,

Or darkling grubs this earthly hole,

In low pursuit:

Know, prudent, cautious, self-controul

Is wisdom's root. (v. 25-30)

Le poème qui clôt le recueil se termine sur une sorte de palinodie, qui frôle l'apostasie, car il contredit bien des valeurs paradées dans les poèmes précédents. Le lecteur est donc amené à réviser toute lecture trop littérale de cette épitaphe inscrite non sur une pierre, mais sur une page. Il est invité à cette entreprise par les hyperboles rhétoriques («wild», v. 16; «quick», v. 20; «keenly», v. 21, 26-27) et sentimentales («drap a tear», v. 6 ; «sigh», v. 12; «the starting tear», v. 17 ; «poor», v. 19), et à voir en elles une forme d'auto-parodie (le poème étant lui-même une réécriture de l'épitaphe placée à la fin du poème de Thomas Gray, «Elegy Written in a Country Churchyard»). Cette interprétation est encouragée par la métaphore filée du sol, de la terre : la «racine» de la sagesse se trouve enfouie dans «ce trou terrestre» (v. 27) : la sagesse résiderait du côté de l'enfer («darkling», au vers 27, est d'ailleurs un adjectif miltonien utilisé dans Paradise Lost, III, 39).

Burns compose aussi des épitaphes plus sérieuses, avec empathie, pour les personnes qui ont compté ou comptent encore pour lui. «Epitaph on my Own Friend and my Father's Friend, Wm. Muir in Tarbolton Mill» (composée en 1784, publiée en 1800) est formée sur un rythme funèbre :

An honest man here lies at rest

As e'er God with his image blest;

The friend of man, the friend of truth,

The friend of age, and guide of youth:

Few hearts like his, with virtue warm'd,

Few heads with knowledge so informed:

If there's another world, he lives in bliss;

If there is none, he made the best of this. 
La grande densité allitérative renforce la structure répétitive qui semble psalmodiée, elle-même soutenue par les distiques rimés. Dès le premier vers, l'accent est mis sur l'honnêteté et le cœur ${ }^{9}$, vertus cardinales selon Burns. Dans l'édition de Kilmarnock, Burns place une série de trois épitaphes positives suivies de trois épitaphes acerbes. Parmi les épitaphes sentimentales, «Epitaph on my Ever-Honoured Father» est consacrée à la figure de son père :

O ye whose cheek the tear of pity stains,

Draw near with pious rev'rence, and attend!

Here lie the loving Husband's dear remains,

The tender Father, and the gen'rous Friend;

The pitying Heart that felt for human Woe,

The dauntless heart that fear'd no human Pride;

The Friend of Man, to vice alone a foe;

For "ev'n his failings lean'd to Virtue's side".

Saturé de vocabulaire sentimental, le poème place au centre le père et le cour; le père étant lui-même le centre organisateur et vital d'un réseau de sociabilité. L'adjectivation et les césures soutiennent le rythme binaire, qui semble être la marque d'un équilibre atteint, ou peut-être obtenu à nouveau, par-delà la perte. La citation finale provient d'un vers d'Oliver Goldsmith : le père littéral est sublimé en un père littéraire.

Les épitaphes «sérieuses» de Burns se caractérisent par un mélange d'ingéniosité et de pathétique, de sentimentalisme. «Epitaph on a LapDog Named Echo» participe de cette veine sentimentale, tout en s'inscrivant dans la tradition classique. Burns évoque la mort d'un chien, comme d'autres auparavant avaient écrit l'épitaphe d'un animal chéri ${ }^{10}$.

In wood and wild, ye warbling throng,

Your heavy loss deplore;

Now, half extinct your powers of song,

Sweet Echo is no more.

Ye jarring, screeching things around,

Scream your discordant joys;

Now, half your din of tuneless sound

With Echo silent lies.

9. Dans «Epitaph for Robert Aiken, Esq.», le poète joue sur l'amphibologie de «heart», à la fois l'organe symptôme de la vie et le symbole de la générosité et de l'humanité : «A warmer heart death ne'er made cold» (v. 4).

10. Laurens cite une «épitaphe d'une sauterelle», une «mort de la mésange» (2007, p. 79, 91), dans la tradition de poèmes en l'honneur du moineau de Lesbie, du perroquet de Corinne, et du chien Peloton dans les feux rustiques de du Bellay. 
Si la première strophe est de facture élégiaque, la deuxième change complètement de tonalité : le chien apporte sa part de chaos sonore dans le monde. Il convient aussi sans doute d'entendre dans le nom du chien une forme de cratylisme : le chien répond à l'appel de son nom, et revient près de son maitre, de même qu'un son lancé contre une paroi rocheuse revient aux oreilles de celui qui l'émet, par le phénomène d'écho. Le lecteur peut se demander si, par-delà l'humour du poème, il n'y a pas un autoportrait et un ars poetica caché dans ce diptyque. Burns se situe dans la tradition tout en s'en démarquant par ses forfanteries provocatrices. Loin du sentimentalisme un peu béat d'un peintre animalier comme Edwin Landseer, Burns sauve son poème du pathétique en rappelant avec véracité le caractère du chien Echo.

Si l'épitaphe est une manière de faire entrer le monde de l'esprit dans le monde des sentiments, ces sentiments ne sont pas toujours dénués de railleries. «Epitaph for Mr William Michie / Schoolmaster of Cleish Parish, Fifeshire» joue avec l'identité du sujet traité, un instituteur (qui n'était pas décédé lorsque Burns composa le poème). Donald Low signale que la cible du poème est probablement John Wilson, l'instituteur et apothicaire de Tarbolton, qui déclenche l'ire de la Mort dans «Death and Doctor Hornbook». Il signale aussi que le poème s'inspire de la traduction d'une épigramme latine imprimée dans Nugae Venales (1663), ce qui indique à la fois la vaste culture de Burns et sa capacité à s'approprier en la «scoticisant» la culture savante :

Here lie Willie Michie's banes ${ }^{\circ}$;

obones

O Satan, when ye tak him,

$\mathrm{Gie}^{\circ}$ him the $\operatorname{schulin}^{\circ} \mathrm{o}^{\prime}$ your weans ${ }^{\circ}, \quad$ ogive, schooling, children

For clever deils ${ }^{\circ}$ he'll mak $^{\circ}$ them!

${ }^{\circ}$ devils, make

L'épitaphe «On Wee Johnie», dont le titre est suivi d'un ô combien traditionnel «Hic jacet wee Fohnie», joue sur une opposition corps/esprit qui renverse le domaine religieux dans le carnavalesque et le rire :

Whoe'er thou art, O reader, know

That Death has murder'd Johnie;

An' here his body lies fu'o low- overy

For saul ${ }^{\circ}$ he ne'er had ony. $\quad{ }^{\circ}$ soul

L'opposition entre les termes sur lesquels repose le trait d'esprit est mise en relief par l'italique. Le dernier vers du quatrain est indiqué par le tiret qui, affilé comme une pointe, mène à la conclusion : le néant («ne’er had ony»). Derrière le raffinement de la syntaxe, les formes brèves de Burns se rapprochent du limerick, comme dans le cas de «On Andrew Turner» : 
In Se'enteen Hunder 'n Forty-Nine,

The Deil gat ${ }^{\circ}$ stuff to mak a swine,

An' coost ${ }^{\circ}$ it in a corner;

${ }^{\circ}$ devil got

${ }^{\circ}$ cast/threw

But wilily he chang'd his plan,

An' shap'd it something like a man,

An' ca'd ${ }^{\circ}$ it Andrew Turner.

${ }^{\circ}$ called

L'épitaphe, texte court qui établit le bilan d'une vie, sert à Burns pour terminer un poème de façon dynamique. «Epitaph for Gavin Hamilton, Esq. », en évoquant «canting wretches», fait référence aux ennemis de Gavin Hamilton, dont faisait partie Holy Willie. Le différend entre eux est mis en scène dans «Holy Willie's Prayer». Burns gratifie Holy Willie de sa propre épitaphe : «Epitaph on Holy Willie», dans laquelle il crée le néologisme «devilship» formé sur «lordship» à propos du représentant religieux. «Epistle to James Smith» a une prolongation dans «Epitaph for James Smith [Epitaph on a Wag in Mauchline] », où, sous l'élégie feinte, le poète subvertit l'avertissement au passant en révélant in fine (in cauda, pourrait-on dire) un trait caractéristique de James Smith :

Lament him, Mauchline husbands a',

He aften ${ }^{\circ}$ did assist ye;

${ }^{\circ}$ often

For had ye staid ${ }^{\circ}$ whole weeks awa’’,

${ }^{\circ}$ stayed, away

Your wives they ne'er had miss'd ye.

Ye Mauchline bairns, as on ye pass

To school in bands thegither ${ }^{\circ}$,

otogether

$\mathrm{O}$ tread ye lightly on his grass,-

Perhaps he was your father!

Parfois, l'épigramme prend l'apparence de la brachylogie : sa concision, la force de l'ellipse, rendent le sens obscur. La pointe jette une lumière rétrospective sur le sens : c'est par exemple le cas de «Monody / On a lady famed for her Caprice». Le poème est constitué de six quatrains en rimes croisées, le dernier étant intitulé «Epitaph». Chacune des cinq premières strophes fonctionne comme une épigramme à la fois reliée aux autres et possédant une certaine autonomie. La première strophe mêle avec intensité l'élégie et la critique :

How cold is that bosom which Folly once fired,

How pale is that cheek where the rouge lately glisten'd;

How silent that tongue which the echoes oft tired,

How dull is that ear which to flatt'ry so listen'd.- (v. 1-4)

L'inversion du sujet et du verbe, l'ellipse du verbe être au vers trois, l'inversion du complément et du verbe dans les vers trois et quatre («the 
echoes oft tired / to flatt'ry so listen'd») rendent l'incipit cryptique. Les hyperbates («that bosom which», «that cheek where»...) prolongent et nuancent la tournure exclamative de chaque vers : elles établissent un parallélisme syntaxique (organisé autour des pronoms relatifs en position centrale) qui met en opposition le début et la fin de chaque vers (par exemple «pale»/ «rouge» au vers 2), tout en recouvrant une autre opposition, chronologique celle-ci, entre le présent et le passé. Au champ lexical de la mort («cold / pale / silent / dull») se combinent les blasons du corps féminin («bosom / cheek / tongue / ear») évoqués sur un mode négatif, comme si la vanité de cette femme (Maria Riddell, avec qui Burns était en froid après l'avoir beaucoup recherchée) était inscrite dans son corps, dans sa nature même. Plus loin, le vers 8, «Thou diedst unwept, as thou livedst unlov'd. - - repose sur le rôle logique de «as», encadré par deux structures syntaxiques semblables («thou» + verbe + forme verbale adjectivée en «un-»), renforcées d'allitérations et d'une rime interne. «As» semble proposer un équilibre entre la mort et la vie, mais il révèle en réalité la similitude des déséquilibres inhérents à la personne critiquée. Ces épitaphes rageuses ou comiques qui culminent sur une sentence ou une pointe sont déjà une forme d'épigramme.

\section{Les épigrammes}

L'épigramme contient souvent un patron rythmique, un quatrain en rimes plates. La pointe qui caractérise le genre a un double but dramatique : elle pique et elle conclut. L'épigramme valorise le fait divers, sous la forme d'une saynète avec un retournement dramatique enchâssé dans la rhétorique. Cette poésie de circonstances est un trait de l'œuvre de Burns, comme l'indiquent les dates ajoutées aux épîtres versifiées à John Lapraik (premier et vingt-et-un avril 1785), les sous-titres de «To a Louse» : «On Seeing One on a Lady's Bonnet at Church» et de «To a Mouse» : "On Turning her up in her Nest with the Plough», ou encore la demi-douzaine de poèmes intitulés «Extempore», dont l'édition est posthume ${ }^{11}$. «Epistle to James Smith» dramatise jovialement l'instant créateur : "Just now I've ta'en the fit o' rhyme» (v. 19).

11. Signalons «An Extemporaneous Effusion / On Being Appointed to the Excise» : «Searching auld wives' barrels, / Ochon [alas], the day! / That clarty bam [dirty yeast] should stain my laurels; / But - what'll ye say! / These muvin' things ca'd [called] wives an' weans [children] / Wad muve the very hearts o’ stanes!» 
Les repas, les banquets sont des occasions de rivaliser d'esprit, et le canon burnsien propose quelques exemples d'épigrammes sympotiques ${ }^{12}$. L'épigramme du touriste, qui est dans la tradition antique (Laurens, 2007, p. 22-23) est aussi façonnée par la circonstance. Mentionnons notamment «Versicles on Sign-Posts», «Verses written on a Window of the Inn at Carron», qui critique l'enfer de la mine, «Epigram at Inveraray», où Burns s'indigne d'avoir été mal reçu dans les Hautes-Terres.

L'épigramme est souvent un substitut verbal du portrait — souvent, aussi, de la caricature, qui laisse au lecteur le soin de conclure. Si Martial recommandait d'«épargner les personnes, [pour] s'en prendre aux vices» (livre X, épigramme 33, v. 10), Burns ne suit pas son conseil :

Among the subjects on which he was accustomed to dwell, the characters of the individuals with whom he happened to meet was plainly a favourite one. The remarks he made on them were always shrewd and pointed, though inclining too much on sarcasm. [...] His wit was ready, and always impressed with the mark of vigorous understanding. (Brown, 1972, p. 8).

Burns ne craint pas d'épingler les hommes d'esprit de son temps, comme Edmund Burke, Adam Smith ou Samuel Johnson. Lecteur attentif de The Theory of Moral Sentiments (1759) d'Adam Smith, Burns composa une épitaphe spirituelle à la mort du philosophe écossais en 1790, «On the Late Death of Dr Adam Smith» :

Death and Hermes of late Elysium made boast,

That each would bring thither what earth valued most;

Smith's Wealth of Nations Hermes stole from his shelf;

DEATH just won his cause - he took of Smith himself.

Burns loue indirectement l'œuvre du penseur, que se disputent la Mort et Hermès, et mêle allègrement deux univers de croyances païennes. Dans «On Mr Burke», Burns s'attaque à Edmund Burke, avec lequel il divergeait sur le plan politique, comme l'indique le sous-titre : «By an Opponent and a Friend to Mr Hastings» :

12. «Epigram on Parting with a Kind Host in the Highlands», «Immediate Extempore, on Being told by W. L. of the Customs Dublin that Commissar Goldie did not seem disposed to push the Bottle», «Epitaph on John Dove, Innkeeper, Mauchline», "Grace before Meat» et «Grace after Meat»; et quelques apocryphes : «At Brownhill Inn», «At Roslinn Inn», et le plus célèbre, connu sous le nom de Selkirk Grace : «Some have meat and cannot eat, / Some cannot eat that want it; / But we have meat and we can eat, / So let the Lord be thankit. » Burns est dans la tradition de Martial : «Son livre n'est pas fait pour être expliqué en classe : compagnon de table et d'orgie, il ne doit paraître qu'à l'heure du souper. » (Laurens, 2007, p. 25) 
Oft I have wonder'd that on Irish ground

No poisonous Reptile ever has been found:

Revealed the secret stands of great Nature's work:

She preserved her poison to create a Burke!

L'attaque ad hominem fonctionne grâce au nom-déclencheur de l'auteur de Reflections on the French Revolution. Prenant prétexte de découvrir le mécanisme de la Nature, le mécanisme réel de cette épigramme est conçue comme un révélateur («revealed», v. 3) de l'identité véritable de Burke; le point d'exclamation est un raccourci typographique d'un «eurêka» sarcastique. De façon similaire, le très bref : «On Johnson's Opinion of Hampden» — «For shame! / Let Folly and Knavery / Freedom oppose: / 'Tis suicide, Genius, / To mix with her foes » — est une modeste réprimande envers le conservateur Samuel Johnson, qui était pour Burns, touché par The Lives of the Poets, un génie égaré, et s'en était pris à John Hampden, une figure radicale de l'époque.

Dans ses études de caractère tracées à l'eau-forte, Burns sait tourner des compliments galants, comme dans «Anna, Thy Charms» (écrit en 1789, publié en 1793):

Anna, thy charms my bosom fire,

And waste my soul with care;

But ah! how bootless to admire,

When fated to despair!

Yet in thy presence, lovely Fair,

To hope may be forgiven;

For sure 'twere impious to despair

So much in sight of Heaven.

qui est d'inspiration similaire à «Epigram to Miss Ainslie in Church / Who was looking up the text during sermon»:

Fair maid, you need not take the hint,

Nor idle texts pursue:

'Twas guilty sinners that he meant,

Not Angels such as you.

Lorsque Burns veut plaire, il pousse l'hyperbole jusqu'au paradoxe (mais la pointe rachète-t-elle la veine courtisane pour autant?) :

"Praise Woman still," his lordship roars,

"Deserv'd or not, no matter?"

But thee, whom all my soul adores,

Ev'n Flattery cannot flatter: 
Maria, all my thought and dream,

Inspires my vocal shell;

The more I praise my lovely theme,

The more the truth I tell. «Complimentary Epigram on Maria Riddell»

Dans la deuxième strophe de «Epigram on Seeing Miss Fontenelle in a Favourite Character», Burns expose sa version du paradoxe sur le comédien — sur la comédienne en l'occurrence :

Wert thou awkward, stiff, affected,

Spurning Nature, torturing art;

Loves and Graces all rejected,

Then indeed thou'd'st act a part. (v. 5-8)

À propos d'une autre jeune femme, Burns utilise la légèreté d'un jeu de mots pour aborder la question, plus sérieuse, du nationalisme écossais :

O had each Scot of ancient times

Been, Jeanie Scott, as thou art;

The bravest heart on English ground

Had yielded like a coward. (v. 9-12)

Mais, comme on a déjà pu le voir, l'épigramme «sur» est souvent une épigramme «contre». Comme Catulle et Martial ${ }^{13}$, Burns enferme la subjectivité et la violence dans le poème court, laissant parler la mauvaise langue, l'esprit de raillerie, et la critique virulente. Après une dispute avec Maria Ridell, Burns se fait cinglant dans «Pinned to Mrs Walter Riddell's Carriage», une épigramme qui devrait la poursuivre comme son ombre :

If you rattle along like your Mistress' tongue,

Your speed will outrival the dart;

But a fly for your load, you'll break down on the road,

If your stuff be as rotten's her heart.

Dans «On a Swearing Coxcomb», Burns prend un malin plaisir à imiter l'accent suffisant et snob de son modèle :

Here cursing, swearing Burton lies,

A buck, a beau, or "Dem my eyes!"

Who in his life did little good,

And his last words were "Dem my blood!"

13. Sur l'épigramme chez Martial et Catulle, voir par exemple Laurens (2007, p. 22-28), et Martin et Gaillard (1990, p. 404-415). 
C'est ce genre d'épigrammes qu'il publie dans l'édition de 1786, en privilégiant la satire des types à la satire des individus, dans «On a Celebrated Ruling Elder» (dont le nom était remplacé par des astérisques dans l'édition originale), et dans une série de trois épigrammes : «Epitaph on a Henpecked Squire», «Epigram on Said Occasion», «Another». Dans cet enchaînement qui propose trois éclairages sur un couple mal assorti, Burns rapproche l'épigramme de la fable, du fabliau médiéval, en plaçant le premier quatrain dans un hors-temps semblable $\mathrm{au}$ «il était une fois» des contes :

As father Adam first was fool'd,

(A case that's still too common,)

Here lies man a woman ruled,

The devil ruled the woman. («Epitaph on a Henpecked Squire»)

Le troisième poème met clairement en parallèle une situation mythologique et une situation contemporaine, alternant grotesque dignifiant (la mégère devient «Queen Netherplace») et burlesque dégradant (le mythe de l'amour sublime est suivi de son équivalent prosaïque) :

One Queen Artemisia, as old stories tell,

When deprived of her husband she loved so well,

In respect for the love and affection he show'd her,

She reduc'd him to dust and she drank up the powder.

But Queen Netherplace, of a diff'rent complexion,

When called on to order the fun'ral direction,

Would have eat her dead lord, on a slender pretence,

Not to show her respect, but - to save the expense! («Another»)

Burns écrit de brefs poèmes dans la tradition populaire des lazzi et des versus ludicri (Laurens, 2007, p. 371-372). Les pasquils - genre dans lequel s'illustre George Buchanan, pour dénoncer la simonie — s'attachent et s'attaquent aux vices personnels des représentants des pouvoirs religieux et temporels. En janvier 1796, le poète d'Ayr compose «The Dean of Faculty», chanson dans laquelle il déplore la défection d'hommes de mérite comme Henry Erskine au profit des loyalistes réactionnaires : "The more incapacity they bring, / The more they're to your liking» (v. 39-40). «Lines on Seeing the Royal Palace at Stirling in Ruins» semble écrit sur un coup de rage :

The injured STEWART line is gone,

A Race outlandish fills their throne;

An idiot race, to honour lost;

Who know them best despise them most.- (7-10) 
Les tétramètres iambiques aux lapidaires distiques contiennent des assertions écrites au vitriol et lancées au visage des héritiers de la Glorieuse Révolution. Des monosyllabes aux dentales hérissées émergent des polysyllabes qui cristallisent l'antagonisme honneur/mépris («honour/ despise»). Nul doute que la densité de l'expression contribue à l'effet de choc qui est produit même sur ses amis :

I have almost given up the excise idea. I have been just now to wait on a great person, Miss -'s friend, Mrs Stewart. - Why will great people not only deafen us with the din of their equipage, and dazzle us with their fastidious pomp, but they must also be so very dictatorially wise? I have been questioned like a child about my matters, and blamed and schooled for my inscription on Stirling window ${ }^{14}$.

Ces rebuffades continues donnent à Burns un sentiment de lassitude qu'il incarne dans «On Politics»:

In politics if thou would'st mix,

And mean thy fortunes be;

Bear this in mind, be deaf and blind,

Let great folk hear and see.

Suite à son repli du monde politique, il se tourne vers Vénus, et dans "I murder hate», il condense sa philosophie de la vie en une courte maxime : «I'm rather pleased to make one more / Than be the death of many ${ }^{15} . »$

L'épigramme est l'occasion de montrer la force de l'esprit du paysan face à la bêtise des puissants (selon le dicton «the pen is mightier than the sword»). Le poète arbore une richesse qui ne se mesure ni en argent, ni en arpents ${ }^{16}$. «Epigram on the Laird of Laggan» résume cette posture :

When Morine, deceas'd, to the Devil went down,

'Twas nothing would serve him but Satan's own crown;

14. Lettre à Agnes McLehose du 27 janvier 1788 (Letters 1, p. 220).

15. Pour courante que soit l'alternative entre les jeux de l'amour et les jeux de la guerre, il est possible de discerner un probable écho des Amours d'Ovide (III, 2) : «Soldat, applaudis Mars! J'ai horreur des combats, / Je n'aime que la paix et, dans la paix, l'amour.»

16. Burns n'épargne en ce domaine pas ses alliés, comme Hugh Blair : «I never respect him with humble veneration; but when he kindly interests himself in my welfare, or, still more, when he descends from his pinnacle and meets me on equal ground, my heart overflows with what is called liking: when he neglects me for the mere carcase of greatness, or when his eye measures the difference of our points of elevation, I say to myself with scarcely an emotion, what do I care for him or his pomp either? ... In my opinion Dr Blair is merely an astonishing proof of what industry and application can do.» (Cité dans Lindsay, 1995, p. 32.) 
"Thy fool's head," quoth Satan, "that crown shall wear never,

I grant thou'rt as wicked, but not quite so clever."

Burns déplore le goût esthétique lamentable des élites, qui sont dans la parure et l'apparence :

Through and through th' inspir'd leaves,

Ye maggots, make your windings;

But O respect his lordship's taste,

And spare his golden bindings. («The Book-Worms»)

Burns feint de prendre la défense du noble philistin en opposant le concret de la reliure dorée et l'abstrait des «pages inspirées». Dans son esprit, nul doute que le livre est un corrélat objectif de l'aristocrate : bientôt dévoré par les vers, il ne restera de lui que le doré. «Epigram on a Country Laird, / not quite so wise as Solomon» souligne que le lord n'est qu'un corps idiot. La précision «country» laisse sous-entendre que son arrogance est inadaptée à sa condition rurale. Cette interprétation est confortée par l'épigramme qui est adjointe à la première, «On Being Shewn a Beautiful Country Seat / Belonging to the same Laird» :

We grant they're thine, those beauties all,

So lovely in our eye;

Keep them, thou eunuch, Cardoness,

For others to enjoy!

Burns s'en prend au corps du lord : après une apparente concession, il dénonce cet individu qui possède des «beautés» dont il ne sait pas jouir du fait de sa puissance esthétique défaillante.

Cette critique des goûts aristocratiques conduit naturellement Burns à mettre l'épigramme au service de la critique d'art. Le quatrain «Epitaph on a Noisy Polemic», publié dans l'édition de Kilmarnock, suppose un art de la parole. L'épitaphé est jugé défaillant. Il est désigné comme «such a bleth'rin bitch» (v. 3). Prenant prétexte de la publication d'une traduction des épigrammes de Martial, qu’il juge déplorable, Burns répond par le même médium. Il donne une leçon au mauvais traducteur, dans «On Elphinstone's Translation of Martial's Epigrams ${ }^{17}$ » :

O Thou whom Poesy abhors!

Whom Prose has turned out of doors!

Heard'st thou yon groan? proceed no further!

'Twas laurel'd Martial calling murther!

17. Lettre à Agnes McLehose du 14 janvier 1788 (Letters 1, p. 206). 
En arbitre du bon goût poétique, le barde d'Ayr répond vertement aux correspondants qui demandent son avis sur leur production littéraire. Un certain Simon Gray était particulièrement insistant, et prolifique. Dans une première réponse, Burns répondit rapidement : «Symon Gray, / You're dull today». Comme Gray lui avait envoyé une deuxième série de poèmes, Burns se contenta d'être aussi bref : «Dullness with redoubled sway, / Has seized the wits of Symon Gray». Comme, enfin, Gray ne le laissait pas en paix, Burns mit les points sur les i : «So, Symon dear, your song I'll tear, / and with it wipe my bum» (cité dans Lindsay, 1995, p. 153-154).

Burns ne critique pas seulement la poésie, il juge aussi les peintres. «To a Painter» se déroule en deux temps. Le premier quatrain est un conseil paradoxal, le deuxième quatrain donne une explication cinglante :

Dear I'll gie ${ }^{\circ}$ ye some advice,

You'll tak ${ }^{\circ}$ it no uncivil:

${ }^{\circ}$ give

You shouldna ${ }^{\circ}$ paint at angels mair ${ }^{\circ}$,

${ }^{\circ}$ take

But try and paint the devil.

To paint an Angel's kittle ${ }^{\circ}$ wark $^{\circ}$,

${ }^{\circ}$ should not, more

Wi' Nick ${ }^{\circ}$, there's little danger:

${ }^{\circ}$ tricky work

You'll easy draw a lang-kent ${ }^{\circ}$ face,

But no sae weel ${ }^{\circ}$ a stranger.-R. B.

${ }^{\circ}$ the Devil

'long-known

${ }^{\circ}$ so well

L'ajout de ses initiales, en signe de défi, indique qu'il n'y a pas dialogue mais duel. Le poème est un art du portrait en abyme. Tout en décrivant le mauvais peintre, Burns établit un rapport homothétique entre le créateur et le sujet traité. Son intérêt pour le portrait, et l'émulation qui existe entre le pinceau et la plume, le stimulent à écrire une épigramme envers un nobliau local : «C[opeland]'s faithful likeness, friend painter, would'st seize? / Keep out Worth, Wit and Wisdom: Put in what you please». Il transpose ces questions sur les rapports entre créateur et créature à l'échelle supérieure, celle de la nature elle-même, dans «On Wm. Graham, Esq., of Mossknowe» :

"Stop thief!" dame Nature call'd to Death,

As Willy drew his latest breath;

How shall I make a fool again?

My choicest model thou hast ta'en. 


\section{Paradoxes}

Le poème court, puisant sa force dans une concision fondée sur l'ellipse, tourne sur lui-même et tend vers l'énigme ou le logogriphe. L'ellipse affûte la pointe dans le même temps où elle met en valeur un paradoxe central. L'épigramme devient le lieu d'une coincidentia oppositorum, rapprochant des termes antinomiques ou appartenant à des domaines opposés de la réalité, tels l'abstrait et le concret. «On Hearing it Asserted Falsehood» est un exemple de ces poèmes-paradoxes, fondés sur une antinomie :

On Hearing it Asserted Falsehood

is expressed in the Rev. Dr Babington's very looks.

That there is a falsehood in his looks,

I must and will deny:

They tell their Master is a knave,

And sure they do not lie.

Les jeux du langage atteignent un nouveau sommet lorsque le poème repose sur le tour de force de ne parler «de rien», comme c'est la cas de «Extempore - To Gavin Hamilton», surnommé parfois par les éditeurs «Stanzas on nothing», et qui commence ainsi :

To you, sir, this summons I've sent,

Pray, whip till the pownie ${ }^{\circ}$ is fraething ${ }^{\circ}$; $\quad{ }^{\circ}$ pony, frothing

But if you demand what I want,

I honestly answer you — naething.-

Ne'er scorn a poor Poet like me,

For idly just living and breathing,

While people of every degree

Are busy employed about - naething. - (v. 1-8)

Sur treize strophes passant en revue l'inanité des actions humaines, y compris de la poésie, avec une intensité pathétique croissante (mais non sans humour grivois, comme dans les strophes 9 et 10), Burns construit une forme de priamel. Dans le priamel traditionnel, à une série énumérative gouvernée par l'anaphore s'oppose un dernier élément qui l'annule. Ici, l'anaphore se mue en une épiphore qui exprime elle-même l'annihilation. «Tam Samson's Elegy» fonctionne de façon similaire : après avoir déploré la mort de son ami chasseur et pris à parti animaux et saisons de cette perte, et rédigé son épitaphe, le poète ajoute une strophe intitulée «Per Contra», qui annule ce qui a été énoncé auparavant: "Tam Samson's leevin'!» La structure logique explose dans la chute.

Quoi de plus spirituel qu'une épigramme? Deux épigrammes, ou davantage, combinées dramatiquement. «Epigrams against the Earl of 
Galloway» combine quatre épigrammes assassines. La série incarne le sérieux, guidé par une ligne clairement tracée, ou un vers épuré («bright [...] line», v. 9) :

What dost thou in that mansion fair?

Flit, Galloway, and find

Some narrow, dirty, dungeon cave,

The picture of thy mind.

No Stewart art thou, Galloway,

The Stewarts'llo were brave;

Besides, the Stewarts were but fools,

Not one of them a knave.

Bright ran thy line, O Galloway,

Thro' many a far-fam'd sire!

So ran the far-famed Roman way,

And ended in a mire.

Spare me thy vengeance, Galloway!

In quiet let me live:

I ask no kindness at thy hand,

For thou hast none to give.

Le franc-parler offensif, qui affleure toujours dans l'épigramme, a conscience de son audace. Et dans la dernière strophe, au lieu d'en appeler à la pitié, le poète termine, non pas tant sur une ultime pointe, mais sur un clou qu'il enfonce.

Si la pointe de l'épigramme contient souvent un renversement de perspective, elle prend aussi parfois l'aspect de la sentence, du proverbe. Au milieu de «The Cotter's Saturday Night», les formules courtes, assertives, personnelles ou empruntées, se multiplient pour contraster, après les pratiques religieuses entre le clergé et les paysans, les mœurs des nobles et celles des paysans :

Princes and lords are but the breath of kings,

"An honest man's the noblest work of God;"

And certes, in fair virtue's heavenly road,

The cottage leaves the palace far behind;

What is a lordling's pomp? a cumbrous load,

Disguising oft the wretch of human kind,

Studied in arts of hell, in wickedness refin'd! (v. 165-171)

L'indignation qui s'exprime par assertion radicale se fait aussi entendre dans «Is There for Honest Poverty [Song - For a' That and a' That] ", dont David Daiches souligne «its effectiveness as a series of slogans» (Daiches, 1981, p. 302). 
La brièveté didactique de l'assertion s'illustre bien dans les proverbes amenés comme par une nécessité mécanique par la trame qui les précède. Le proverbe peut être humoristique ("heaven can boil the pot, / Tho' the devil piss in the fire», dans «The Dean of Faculty», v. 15-16) ou audacieux, comme c'est le cas du final de «Love and Liberty [The Jolly Beggars]» :

Life is all a variorum,

We regard not how it goes;

Let them cant about decorum,

Who have character to lose. (v. 288-291)

et du refrain final :

A fig for those by law protected!

Liberty's a glorious feast!

Courts for cowards were erected,

Churches built to please the priest. (v. 297-300)

Ces proverbes nouvellement forgés notent les contradictions sans les résoudre, restituant linguistiquement toute la violence sociale des rapports de classes, dont «To a Louse» donne une version plus douce, presque badine en comparaison: «O wad some Power the giftie gie us / To see oursels as ithers see us!» (v. 43-44). En revanche, «Address to the Unco Guid, or the Rigidly Righteous» est aussi une machine de guerre littéraire contre les bien-pensants. Burns commence par se mettre lui-même en avant, sous la forme d'une exergue qui contient une paraphrase versifiée par ses soins d'un extrait de l'Ecclésiaste. Il entend ainsi montrer sa connaissance du texte biblique, et sa familiarité même, dans la mesure où il ne craint pas de le traduire et de le reformuler, c'est-à-dire enfin de l'interpréter. Cette mise en scène est une première escarmouche dans le poème polémique qui suit et qui s'en prend à ce qu'on appellerait les «grenouilles de bénitier». Chaque strophe est une épigramme avec pointe, dans un dialogue, qui est un discours de défense («defences», v. 14). Burns, en chemin, modifie le proverbe «to err is human» en «to step aside is human", donnant par là l'idée que l'existence se déroule selon un pas de danse, et peut-être qu'il y aurait une esthétique de l'erreur. Dans cette joute de parole biblique contre parole biblique, et de proverbe contre proverbe, Burns joue de ce que «le proverbe généralise la référence» en opérant «une transformation de l'indéfini à l'universel», comme le remarque Zumthor (1976, p. 316), retournant la force parémiologique d'assertion à l'envoyeur, dont la seule qualité est, au total, « [a] better art o' hidin» (v. 24). Il conclut en sermonnant les faiseurs de sermons : 
Who made the heart, 'tis $H e$ alone

Decidedly can try us,

He knows each chord, its various tone,

Each spring, its various bias:

Then at the balance let's be mute,

We never can adjust it;

What's done we partly may compute,

But know not what's resisted. (v. 57-64)

Dans ses lettres, il dénonce avec vigueur «a presbyterian sourness, an hypocritical severity», dont il se défend «when I survey my less regular neighbours ${ }^{18}$. Il y a beaucoup de feintes et de jeux avec la convention dans cette pratique de différentes formes brèves chez Burns. Est-ce un jeu gratuit, comme chez Martial («lusus» [Laurens, 2007, p. 24-25])? Il n'en est rien. Sous l'apparent badinage artistique, sur lequel l'esprit ne ferait que glisser, Burns entend redresser les torts, redonner de la mesure ${ }^{19}$, régler des comptes, comme dans la dernière strophe de "Address to the Unco Guid». Les vérités familières reçoivent un tour neuf et aiguisé par une image en raccourci, par un court-circuit rhétorique, offrant un contraste inattendu, une réalité antinomique, au moyen de parallélismes, de chiasmes, de symétries. Les formes brèves sont propices aux retournements de perspective : entre l'épigraphe et le poème, entre les tons (de l'épitaphe sérieuse à l'épitaphe moqueuse), entre le début et la fin d'une épigramme. Cette perspective évolutive est dramatisée dans des poèmes plus longs comme «To the Unco Guid». Par la concision, l'allitération ou la répétition, la forme brève acquiert la force percutante de la persuasion, et possède un impact psychologique. Par un renversement qui plairait à Burns, le stylet originaire de l'épigramme, passé de la pierre à la page, s'est fait style, sans perdre son tranchant. L'épigramme est un médium concentré pour faire passer les vérités du poète. Une chose est certaine : l'épigramme a le goût de l'homme — «hominem sapit», dit Martial dans ses Épigrammes (Laurens, 2007, p. 26).

18. Lettre du 4 janvier 1788 à Agnes McLehose (Letters 1, p. 195).

19. «Epigram on Mr James Gracie» : «Gracie, thou art a man of worth, / O be thou Dean for ever! / May he be damned to hell henceforth, / Who fauts thy weight or measure!» 


\section{Références bibliographiques}

Brown Raymond L. (ed.), Robert Burns's Tour of the Borders, Ipswich, The Boydell Press, 1972.

Bunns Robert, The Poems and Songs of Robert Burns, Oxford, Oxford University Press, 1968.

Daiches Robert, Robert Burns, Édimbourg, Spurbooks, 1981.

Laurens Pierre (éd.), Anthologie de l'épigramme, de l'Antiquité à la Renaissance, Paris, Gallimard, 2007.

Lindsay Maurice, The Burns Encyclopedia, Londres, Robert Hale, 1995.

Martial, Épigrammes, Paris, Arléa, 2001.

Martin René et Gaillard Jacques, Les genres littéraires à Rome, Paris, Nathan, 1990.

PoréE Marc, «Poétique d'une forme brève : les proverbes de l'enfer blakiens », Études Anglaises, vol. 48, n 4, 1995, p. 395-406.

Ross Roy G. (ed.), The Letters of Robert Burns, Oxford, Oxford University Press, 1985, 2 volumes. [Letters]

SAIId Suzanne, Trédé Monique et Le Boullouec Alain, Histoire de la littérature grecque, Paris, Presses universitaires de France, 1997.

Zumthor Paul, «L'épiphonème proverbial», Revue des Sciences humaines, $\mathrm{n}^{\circ} 163,1976$, p. 313-328. 